American Journal of Pharmaceutical Education 2018; 82 (9) Article 7046.

\title{
COMMENTARY
}

\section{Reflections on 500 Call-in Radio Shows}

\author{
Allison Mari Dering-Anderson, PharmD \\ University of Nebraska Medical Center College of Pharmacy, Omaha, Nebraska \\ Submitted March 1, 2018; accepted June 7, 2018; published November 2018.
}

\begin{abstract}
Pharmacy educators, whether in didactic classes, laboratory settings, or experiential opportunities, search for ways to incorporate "real life" patient questions and concerns into the educational process. This practice not only enhances the educational opportunities for students, it also prepares them for questions and concerns that they will inevitably face as practicing professionals. This commentary describes listener calls from 500, live, call-in radio shows. There is no accurate way to directly assess information that patients do not know or understand. The author suggests using commonly asked pharmacy-based questions, directly from the public, as a proxy to identify the information most desired by patients. The author assumes that radio callers are not calling to ask questions when they already know the answers. Thus, having identified the information most desired by radio-show callers, pharmacist educators, no matter the setting, can be provided with a ready-made resource that lists the most common concerns from over 6,000 radio callers. The author recommends educators use this resource and apply real patient questions and concerns during the educational process, furthermore, opportunities to use this resource are provided.
\end{abstract}

Keywords: patient counseling, pharmacy education, patient communication

I pose a simple question, "How do we know what our patients don't know?" A corollary to this question is, "Are we teaching our students to provide the information patients most need?" As I was preparing for my $500^{\text {th }}$ call-in radio show, by reviewing notes from the previous 499 shows, all tracked on a spreadsheet, I was struck with the notion that perhaps I had helpful information in these nearly 6,000 questions. I wondered, what did callers ask about most often? Could this information be helpful in planning lectures or simulated experiences? Should I use this information in planning examination questions and patient counseling situations? Is this a way to get at the answer to the question, How do we know what our patients don't know?

As pharmacists, we understand that we will never know for certain what our patients don't know. Likewise, we are aware that we have no reliable way to gauge all that our patients don't know or don't understand. McHorney and colleagues reported on how to predict who will be adherent to prescribed medicines. ${ }^{1}$ McHorney's Adherence Estimator considers the patient's belief in the ability of the drug to help with the diagnosed condition and patient concerns about the medication. Neither the questions considered by McHorney, nor the resulting estimation tool assists pharmacists in determining points of confu-

Corresponding Author: Allison Mari Dering-Anderson, University of Nebraska College of Pharmacy, 986145 Medical Center, PDD 2055. Omaha, NE 68198-6145. Tel: 402-5526816. E-mail: Ally.DeringAnderson@UNMC.edu sion or lack of understanding for the patient. We have a variety of scales and other measurements, but we don't have the ability to say, "so, tell me what you don't know."

I propose to use the subject of phone calls to a radio show to discuss those questions and concerns most commonly raised. This information has been collected over a 20year span; during a live, call-in, radio show in the Midwest. KFOR Radio station, located in Lincoln, Nebraska and currently owned by Alpha Media has broadcast, for nearly three decades, a weekday morning show called "Problems \& Solutions." Problems \& Solutions is hosted by a two-time Marconi winner, Cathy Blythe. ${ }^{2,3}$ The Marconi is the highest award in radio broadcasting, like the Tony for Broadway productions. This reflection was stimulated by my preparation for the production of the $500^{\text {th }}$ show wherein I answer questions from the public.

I have tracked each caller's question, over the years, and have sorted them into descriptive categories; identifying the drug and the nature of the question. It is not my intent to provide an exhaustive listing of all questions, nor even to try to turn these observations into traditional research, rather I will present the most commonly asked questions from several classifications. The intent is to guide precepting pharmacists and pharmacy faculty by identifying those topics, self-identified by patients, which may help us learn what patients don't know.

In 2015, Porney and colleagues offered a qualitative review of patients' engagement in their health care. ${ }^{4}$ An exciting idea and a laudable goal until we recognize that 


\section{American Journal of Pharmaceutical Education 2018; 82 (9) Article 7046.}

patients don't always understand or retain the information we provide to them. We teach and practice patient counseling, we stress patient-friendly language, but we often fail to realize that the information needs to be presented at exactly the time the patient is open to learning it. The most common question asked, in 20 years and 500 shows, is a variant of, "When should I take my thyroid supplement?" While I am hopeful that we have garnered new listeners over the years, for this question to be the most commonly asked question, highlights that the information, no matter how basic, isn't retained by the listeners until it is specific to them. This simple concept - meet the patients where they are - is a hallmark of motivational interviewing, for ambivalent patients or those who are resisting a suggested change. ${ }^{5}$ It is my belief that the people who call the Problems \& Solutions radio show are neither ambivalent, nor change averse; rather, they are looking for a quick answer to a question that they are uncomfortable asking of another health professional or in another setting. The patients and listeners filter the show through the lenses of their lives and their needs. I believe there is a component of reluctance to say, "I know you've told me this before, but I've forgotten" in the calls. There is also a component of "when last you discussed this, I wasn't in a place where the information was relevant to me." I further believe that knowing the subject of these calls will help us to educate, enforce and reinforce the messages that are most often asked for.

\section{Dose Timing}

The most common question is a dose timing question, "When should I take my thyroid supplement?" Other common dose timing questions include: "Do I have to take my statin at bedtime?" and "May I take all of these at the same time?" These questions serve to highlight the need for explicit timing information on prescription labels, "Take when you go to bed" as opposed to "Take 1 in the evening" or worse, "Take 1 daily." The questions may also help to stress the importance, to a sector of our patient population, of convenience packaging services offered by many pharmacies. These questions pose opportunities for realistic assignments in dispensing (appropriate labels), in product preparation (convenience packaging alternatives), or in pharmacy law (May the original patient instructions be altered to improve patient understanding?). They also highlight information that must be accurately transmitted to patients during simulated and real counseling sessions.

\section{Nonprescription Drugs}

The most commonly asked nonprescription drug questions are "which calcium supplement should I take carbonate or citrate salt?" and "how much daily dosage of calcium should I take?" This may present an opportunity for a "shelf talker" offering pharmacist assistance in making these decisions and answering any questions. If we, as pharmacy educators, are unaware of the frequency of these questions, we may miss an opportunity to include this discussion in nondispensing services, pharmacy management, or product merchandising. These questions also highlight that patients who hear reports in mass media are unable to apply them to their own situation without assistance. Conflicting information about calcium supplements and cardiac risk when presented in the mass media, serve to concern and frighten patients. ${ }^{6-8}$

The second most common group of nonprescription drug questions focus on vitamins. Questions about vitamin $\mathrm{C}$ and viral infections and multivitamin supplements were the leading contributors to this category. These questions point to the potential need for collaboration between pharmacists and dieticians and patients, not only the traditional patients with diabetes or congestive heart failure who are often associated with this collaboration. While the health benefits of vitamins can be controversial (some believe they are beneficial and some don't), there is no controversy when it comes to the value of getting vitamins and minerals from a good diet before considering supplements. ${ }^{9}$ While pharmacists are able to answer basic nutrition questions, partnering with a nutrition expert may benefit patients and address their confusion. Our students will benefit from being taught by diet and nutrition experts.

\section{Vaccines}

Vaccine questions are not, as one might expect, seasonal. This is due, in part, to questions concerning vaccines not being restricted to influenza during the traditional season. The type of vaccines most often asked by my callers are pneumococcal vaccines, herpes zoster vaccines, and influenza vaccines. Many callers ask about the appropriate timing of vaccines, the need for revaccination, and the safety of vaccines. The clear recognition that a pharmacist was able to answer these questions is proof that the callers recognize pharmacists as approachable experts in the field of vaccines and vaccine preventable diseases. It is also clear that while callers are familiar with childhood vaccine recommendations, many callers are not familiar with adult vaccine recommendations. For pharmacies that rely on patients requesting vaccine, this is an indication that there are likely many more patients who could benefit from a pharmacist reviewing their vaccination history or a pharmacist administering the vaccine. Information on adult vaccine recommendations should be provided to patients with an identified risk (ie, offering hepatitis vaccine to a patient receiving insulin or offering pneumococcal vaccine to a known smoker), and offering general guidelines to all 


\section{American Journal of Pharmaceutical Education 2018; 82 (9) Article 7046.}

patients throughout the year to promote vaccination services. In October 2001, an entire radio show was dedicated to discussing anthrax. There were multiple questions about anthrax vaccine during that show and continuing through the fall of 2001. Because of the national crisis on mailed anthrax spores, these multiple calls are acknowledged here. ${ }^{10}$ It is noteworthy that the only calls about anthrax vaccine occurred between October 2001 and January 2002.

\section{Disease States}

The most commonly discussed chronic condition is pain. Recent questions focus on concern about long-term use of opiates and opiate-like drugs. Over the entire span of 6,000 shows, questions about chronic pain, pain relief, and pain therapies have been constant. While the question specifics are often influenced by current events, the topic of pain never wanes. Pharmacists must be prepared to answer questions about pain in an informed and empathetic fashion. Often, callers want someone to listen to the story, not necessarily expecting a solution, but simply to listen and empathize. Pharmacists should anticipate questions about dosage, drug-to-drug interactions, potential for substance abuse problems, and fear.

Some of the most challenging questions center on dietary supplements claiming to treat pain where there is no evidence to guide decision making; no evidence of efficacy and no evidence of danger. When there is published information on danger, the answers are easy, but keeping up with that information can be overwhelming. ${ }^{11}$ In addition to selecting learning opportunities that focus on ambulatory pain management, lifelong learning skills are an important outcome in pharmacy education. Helping our students and preceptors identify mechanisms to keep informed with frequently requested information may be highlighted using these kinds of patient questions.

The second most common conditions are those questions in dermatology. This category is highlighted here because this is the category where callers would offer solutions to other callers at the highest rate. This is a heterogeneous category, covering: hair loss, brittle nails, dry and cracked heels, and seasonal questions about biting insects. In most instances, I do not believe that calling a faceless pharmacist on the radio, was the first health care encounter for these patients. Rather, I believe these calls represent frustrations in an inability to find solutions elsewhere. Two important teaching concepts are illuminated through the dermatologic questions: nonprescription drugs are the mainstay of some therapies and should not be overlooked in content planning, and patients will have encounters with pharmacists relying on information from some very questionable sources. It may be important to ask where the information the patient is citing originated. "Old wives cures" mean that these women lived long enough to be old and kept their husbands alive long enough for them to be old wives, as opposed to old widows; but many of these remedies are the antithesis of scientific. Finding a way to tactfully make another recommendation is a skill to be honed and practiced. Each of these points offers opportunities for teaching and skills enhancement in didactic and experiential programs.

\section{Drug and Food}

Removing the timing of thyroid supplements from this section, the two most common drug and food questions are: "Will taking probiotics help with anticipated side effects of antibiotics?" and "Does this interact with grapefruit?" Patients asking these questions want specific and personalized information - they want to know which probiotic, in what dose, at what time and for how long. Pharmacists in all settings should be ready to answer each of these questions, including having a ready recommendation when appropriate. Patients concerned about grapefruit interactions often misunderstand that this is not a timing issue. These patients know that if they take a bulking laxative, they must take it separately from an oral anticoagulant by 2 hours and they expect that separating the drug in question from grapefruit by 2 hours will be sufficient. It is difficult to explain the inhibition of a cytochrome enzyme when a patient is used to understanding physical binding or practical stomach protection when taking drugs with food. The FDA offers resources to help explain this CYP3A concern, such as the reference found as Figure $1,{ }^{12}$ but this resource isn't helpful if it isn't available when talking to the patient. Perhaps precepting pharmacists should plan to have copies of this, or a similar resource, available for those drugs most likely to be involved in these interactions and provide them proactively to patients.

\section{Final Considerations}

There may be similar access to public comments and questions nearer to other pharmacy schools and colleges, using them will help to highlight the most common questions in those regions. No attempt has been made to compare these experiences with the experiences of pharmacists in other regions of the country, in other countries in the world, or in populations significantly different from the KFOR listening and calling audiences. The primary characteristics of the listening audience are: female, white, $>50$ years of age, with some post-high school education. It is undetermined if the calling audience is representative of the listening audience. Even with these limitations, an evaluation of the nearly 6000 questions from the public does serve to highlight broad 


\section{American Journal of Pharmaceutical Education 2018; 82 (9) Article 7046.}

When drugs are swallowed, they may be broken down (metabolized) by enzymes and/or absorbed using transporters in cells found in the small intestine. Grapefruit juice can cause problems with these enzymes and transporters, causing too much or too little drug in the body.

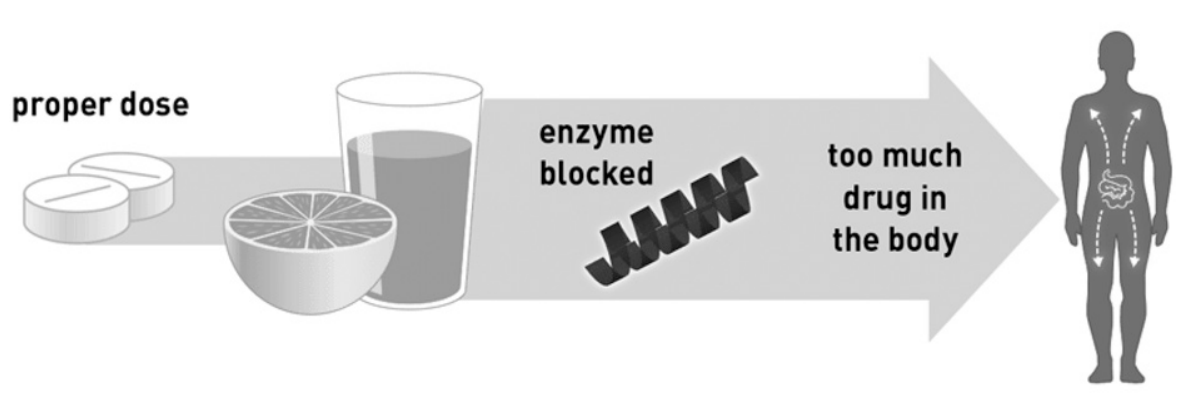

Some drugs, like statins used to lower cholesterol, are broken down by enzymes. Grapefruit juice can block the action of these enzymes, increasing the amount of drug in the body and may cause more side effects.

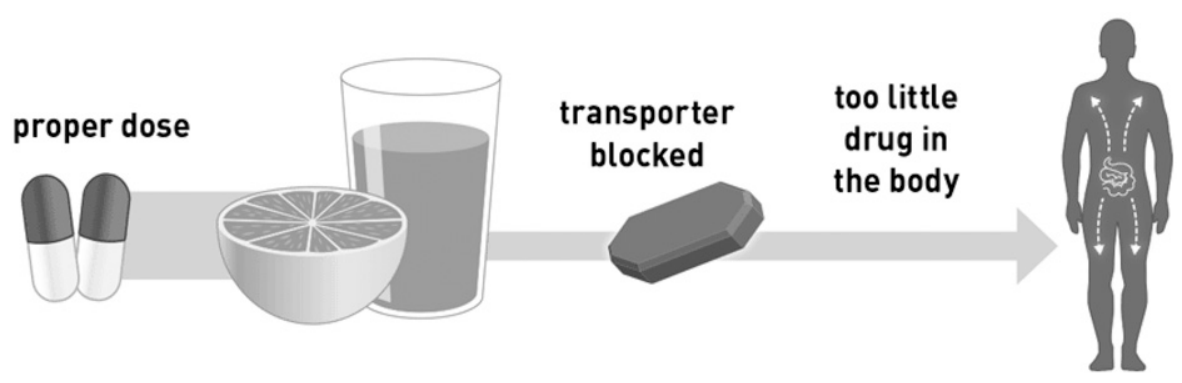

Other drugs, like Allegra (fexofenadine) used to treat allergies, are moved by transporters into the body's cells. Grapefruit juice can block the action of transporters, decreasing the amount of drug in the body and may cause the drug to not work as well.

Info-Graphic courtesy of FDA Consumer Health Information, used with permission

Figure 1. How Grapefruit Juice Affects Some Drugs ${ }^{12}$.

categories of patient requests for information. Each of these categories provide ample opportunity for education, for skills development, as well as knowledge acquisition, and for concept reinforcement while providing patient counseling, and while conducting medication therapy management (MTM) reviews.

Finding practical examples and common questions and concerns isn't always an easy task. I am advocating that using real questions from the local public can add realism to our educational process. Further, we may find that we aren't teaching those concepts most often requested by members of our patient base.

\section{REFERENCES}

1. McHorney CA. The adherence estimator: a brief, proximal screener for patient propensity to adhere to prescription medications for chronic disease. Curr Med Res Opin. 2009;25(1):215-238.
2. WSB, Paul Harvey among Marconi winners. RadioWorld. Sept. 16, 2002. https://www.radioworld.com/news-and-business/wsb-paulharvey-among-marconi-winners. Accessed February 16, 2018.

3. Korbelik J. KFOR's morning team wins prestigious radio award. Lincoln Journal Star. September 22, 2005.

4. Pomey MP, Ghadiri DP, Karazivan P, Fernandez N, Clavel N. Patients as partners: a qualitative study of patients' engagement in their health care. PLoS One. 2015;10(4):e0122499.

5. Berger BA, Bertram CT. Motivational interviewing and specialty pharmacy. J Manag Care Spec Pharm. 2015;21(1):13-17.

6. Calcium and heart disease: what is the Connection? January 2017, Harvard Heart Letter. https:/www.health.harvard.edu/heart-health/calciumand-heart-disease-what-is-the-connection. Accessed February 16, 2018. 7. Anderson JJ, Kruszka B, Delaney JA, et al. Calcium intake from diet and supplements and the risk of coronary artery calcification and its progression among older adults: 10-year follow-up of the multi-ethnic study of atherosclerosis (MESA). J Amer Heart Assoc. 2016;5(10):e003815. 8. Bolland M, Grey A, Reid I. Calcium supplements and cardiovascular risk: 56 years on. Ther Adv Drug Saf. 2013;4(5):199210. 


\section{American Journal of Pharmaceutical Education 2018; 82 (9) Article 7046.}

9. Vitamin supplements: healthy or hoax? American Heart Association. 2015. http://www.heart.org/HEARTORG/Conditions/ Vitamin-Supplements-Healthy-or-Hoax_UCM_432104_Article. jsp\#.WpXZDRPwZ24. Accessed February 27, 2018.

10. United States Department of Justice Press Release \# 10-166. February 19, 2010, and attachments.
11. Ulbricht C, Costa D, Dao J, et al. An evidence-based systematic review of kratom (Mitragyna speciose) by the Natural Standard Research Collaboration. J Dietary Suppl. 2013;10(2):152-170. 12. FDA consumer update: grapefruit juice and some drugs don't mix; www.fda.gov/forconsumers/consussmerupdates/ucm 292276. htm. Accessed February 17, 2018. 